\title{
ON SUBGROUP COMMUTATORS
}

\author{
M. F. NEWMAN
}

1. Introduction and notation. The aim of this note is to present a generalization of two results; one of Hobby and Wright (Theorem 1 of [3]) and the other of Janko [4].

The symbols $\leqq,<, \triangleleft$ mean, in turn, "is a subgroup of", "is a proper subgroup of", "is a normal subgroup of". A subgroup $S$ is subnormal in a group $G$ if there are subgroups $S_{1}, \cdots, S_{m}$ of $G$ such that $S \triangleleft S_{1} \triangleleft \cdots \triangleleft S_{m} \triangleleft G$; the length of the shortest such chain is the subnormal length of $S$ in $G$ (thus proper normal subgroups have subnormal length 1). If $a, b, c$ are elements of $G$, then $a^{b}=b^{-1} a b$, $a^{-b}=\left(a^{-1}\right)^{b},[a, b]=a^{-1} a^{b}$, and $[a, b, c]=[[a, b], c]$. If $A, B, C$ are subsets of $G$, then the subgroup generated by $A$ is denoted $\{A\}$; the normal closure of $A$ in $G$ is denoted $A^{G}$; the subgroup generated by all commutators $[a, b]$ where $a \in A, b \in B$ is denoted $[A, B]$; and the iterated commutator $[[A, B], C]$ is abbreviated to $[A, B, C]$. The $n$th $(n$ a positive integer) term $[G, \cdots, G]$ ( $n$ repetitions of $G$ ) of the lower central series of $G$ is denoted $\gamma_{n}(G)$, the Frattini subgroup of $G$ by $\Phi(G)$, and the subgroup generated by the $n$th powers of elements of $G$ by $\beta_{n}(G)$.

(1.1) TheOREM. Let $n$ be a positive integer. Let $G$ be a nilpotent group in which $\gamma_{n}(G)$ has the following properties:

(a) for every prime $p$ the Sylow p-subgroup has finite exponent, and

(b) the factor group by the periodic subgroup (i.e., the subgroup generated by and consisting of the elements of finite order) is finitely generated.

If $H$ is a subgroup of $G$ such that $\gamma_{n}(H)<\gamma_{n}(G)$, then

$$
\gamma_{n}\left(H \gamma_{2}(G) \beta_{p}(G)\right)<\gamma_{n}(G)
$$

for some prime $p$.

Since $\gamma_{2}(G) \beta_{p}(G)$ is (obviously) the intersection of all the normal subgroups of index $p$ in $G$, an immediate corollary of this is:

(1.2) Corollary. If $n, G, H$ are as in Theorem 1.1, then

$$
\gamma_{n}(H \Phi(G))<\gamma_{n}(G) .
$$

It is natural to ask to what extent this result is best possible. Simple examples (which are omitted) show that the conclusion of the

Received by the editors July 30, 1962. 
theorem does not hold when the hypotheses are weakened in any obvious way.

The paper of Hobby and Wright has two mistakes. The first occurs in the proof of their Lemma 1. The referee informs me that Hobby and Wright sent him a correction of this with an offprint. The proof of Lemma 2.1 below is the referee's which he based on Hobby-andWright's correction; my original proof was based on a slightly different inductive step. The second mistake was recently pointed out and corrected by Hill [2].

The results on subgroup commutators which are used in the proof, in $\$ 3$, of Theorem 1.1 are derived in $\$ 2$. By using more refined commutator calculations it is possible to put Hill's Theorem B in the same setting. (This will not be done here.)

I am greatly indebted to the referee; this note owes much to his constructive comments, for these I thank him. I am indebted to Dr. Janko for drawing my attention to [4].

2. Commutator calculations. The proof of Theorem 1.1 is made to depend on some results on subgroup commutators. These are derived here from the well-known element commutator identities:

$$
\begin{aligned}
{[a, b] } & =[b, a]^{-1}, \\
{[a b, c] } & =[a, c][a, c, b][b, c], \\
{[a, b c] } & =[a, c][a, b][a, b, c], \\
{[b, c, a] } & =\left[a, b^{-1}, c^{-1}\right]^{-b c}\left[c^{-1}, a^{-1}, b\right]^{-a c},
\end{aligned}
$$

(see, for example, [1, p. 150]).

If $A, B$ are subgroups of a group $G$, then, using (3), an easy induction shows that

$$
\prod_{i=1}^{r}\left[a, b_{i}\right]^{-1}\left[a, \prod_{i=1}^{r} b_{i}\right] \in[A, B, B]
$$

for all $a \in A$, all $b_{i} \in B$, and all positive integers $r$. Hence

$$
\prod_{i=1}^{r}\left[a, b_{i}\right]^{-k}\left[a, \sum_{i=1}^{r} b_{i}^{k}\right] \in[A, B, B]
$$

for all $a \in A$, all $b_{i} \in B$, and all positive integers $r, k$.

If $A, B, C$ are subgroups of a group $G$, then the following subgroup commutator relations are immediate consequences of (1)-(5) above:

$$
\begin{aligned}
{[A, B] } & =[B, A], \\
{[A B, C] } & \leqq\{[A, C],[B, C],[A, C, B]\},
\end{aligned}
$$




$$
\begin{aligned}
{[A, B C] } & \leqq\{[A, B],[A, C],[A, B, C]\}, \\
{[B, C, A] } & \leqq[A, B, C]^{\sigma}[C, A, B]^{a}, \\
{\left[A, \beta_{k}(B)\right] } & \leqq \beta_{k}([A, B])[A, B, B]^{a} .
\end{aligned}
$$

(2.1) LemMA. If $H$ is a subgroup of a group $G$, then

$$
\left[G, \gamma_{n}(H)\right]^{G} \leqq\left[H, \gamma_{n}(G)\right]^{G}
$$

for every positive integer $n$.

Proof. Let $N=\left[H, \gamma_{n}(G)\right]^{a}$. It suffices to prove

$$
\left[\gamma_{n-m}(G), \gamma_{m+1}(H)\right] \leqq\left[\gamma_{n-m+1}(G), \gamma_{m}(H)\right]^{G} N
$$

for all $m \in\{0,1, \cdots, n-1\}$ because then the result follows by an easy induction. Now, using (6),

$$
\begin{aligned}
{\left[\gamma_{n-m}(G), \gamma_{m+1}(H)\right] } & =\left[\gamma_{m}(H), H, \gamma_{n-m}(G)\right] \\
& \leqq\left[H, \gamma_{n-m}(G), \gamma_{m}(H)\right]^{G}\left[\gamma_{n-m}(G), \gamma_{m}(H), H\right]^{G}, \text { by }(9), \\
& \leqq\left[\gamma_{n-m+1}(G), \gamma_{m}(H)\right]^{G} N
\end{aligned}
$$

using (6) and the fact that $\left[\gamma_{n-m}(G), \gamma_{m}(G)\right] \leqq \gamma_{n}(G)$ (see, for example, $[1$, p. 156]).

It is perhaps of interest to record the following consequence of Lemma 2.1 which generalizes Theorem 2 of [3].

(2.2) THEOREM. If $H$ is a subgroup of a group $G$ and $\gamma_{n}(H)=\gamma_{n}(G)$ for some positive integer $n$, then $\gamma_{n+1}(H)=\gamma_{n+1}(G)$.

The proof of this is based on the following simple and presumably known generalization of the well-known fact that every subgroup of a nilpotent group is subnormal.

(2.3) Lemma. If $H$ is a subgroup of a group $G$ and $\gamma_{n}(G) \leqq H$ for some positive integer $n$, then $H$ is subnormal in $G$.

Proof. Let $H=H_{0} \leqq H_{1} \leqq \cdots$ be an ascending sequence of subgroups of $G$ such that $H_{r}$ is the normalizer of $H_{r-1}$ in $G$ for all $r$. Since $\gamma_{n}(G) \leqq H$, it follows that $\gamma_{n-1}(G) \leqq H_{1}$ and, by an easy induction, that $G=H_{n-1}$; i.e., $H$ is subnormal in $G$.

Proof of 2.2. By Lemma 2.3, $H$ is subnormal in $G$. The proof is by induction on subnormal length.

Let $N$ be a normal subgroup of $G$ such that $\gamma_{n}(N)=\gamma_{n}(G)$, then $\gamma_{n+1}(G)=\left[\gamma_{n}(N), G\right] \leqq\left[N, \gamma_{n}(G)\right]$ (by Lemma 2.1) $\leqq \gamma_{n+1}(N)$ and so $\gamma_{n+1}(G)=\gamma_{n+1}(N)$ because, trivially, $\gamma_{n+1}(N) \leqq \gamma_{n+1}(G)$. 
Let $s$ be an integer greater than 1 and suppose the theorem has been proved for all subgroups of $G$ whose subnormal length is less than $s$. Let $K$ be a subgroup of $G$ with subnormal length $s$ and such that $\gamma_{n}(K)=\gamma_{n}(G)$. Let $L$ be a subgroup of $G$ which has subnormal length less than $s$ and in which $K$ is normal, then $\gamma_{n}(K)=\gamma_{n}(L)$ $=\gamma_{n}(G)$. Hence, by the inductive hypothesis, $\gamma_{n+1}(L)=\gamma_{n+1}(G)$; and $\gamma_{n+1}(K)=\gamma_{n+1}(L)$ because $K \triangleleft L$. Therefore the theorem is true for all subnormal subgroups and the proof is complete.

\section{Proof of Theorem 1.1.}

(3.1) Lemma. If $H$ is a subgroup of a nilpotent group $G$ and if $\gamma_{n}(H)<\gamma_{n}(G)$ for some positive integer $n$, then $\gamma_{n}(H) \gamma_{n+1}(G)<\gamma_{n}(G)$.

Proof. Since every subgroup of $G$ is subnormal (Lemma 2.3), an induction on subnormal length proves the result.

Let $N$ be a normal subgroup of $G$ such that $\gamma_{n}(N)<\gamma_{n}(G)$, then $\gamma_{n}\left(G / \gamma_{n}(N)\right)=\gamma_{n}(G) / \gamma_{n}(N)$ is nontrivial and so, since every factor group of $G$ is nilpotent, $\gamma_{n+1}\left(G / \gamma_{n}(N)\right)<\gamma_{n}\left(G / \gamma_{n}(N)\right)$. Hence $\gamma_{n+1}(G) \gamma_{n}(N) / \gamma_{n}(N)<\gamma_{n}(G) / \gamma_{n}(N)$ and therefore $\gamma_{n+1}(G) \gamma_{n}(N)$ $<\gamma_{n}(G)$.

Let $s$ be an integer greater than 1 and suppose the lemma has been proved in all nilpotent groups for all subgroups whose subnormal length is less than $s$. Let $K$ be a subgroup which has subnormal length $s$ in $G$ and is such that $\gamma_{n}(K)<\gamma_{n}(G)$. Let $L$ be a normal subgroup of $G$ in which $K$ has subnormal length less than $s$. If $\gamma_{n}(L)<\gamma_{n}(G)$, then $\gamma_{n}(L) \gamma_{n+1}(G)<\gamma_{n}(G)$ and, a fortiori, $\gamma_{n}(K) \gamma_{n+1}(G)<\gamma_{n}(G)$. If $\gamma_{n}(L)=\gamma_{n}(G)$, then $\gamma_{n}(K)<\gamma_{n}(L)$ and so $\gamma_{n}(K) \gamma_{n+1}(L)<\gamma_{n}(L)=\gamma_{n}(G)$ by the inductive hypothesis. But $\gamma_{n+1}(L)=\gamma_{n+1}(G)$, by Theorem 2.2, so $\gamma_{n}(K) \gamma_{n+1}(G)<\gamma_{n}(G)$ as required.

Proof of 1.1. Since $\gamma_{n}(G)$ is nilpotent, it is easy to verify that every factor group of $\gamma_{n}(G)$ has properties (a) and (b) of the statement of the theorem. It follows, using Lemma 3.1, that $\gamma_{n}(G) / \gamma_{n}(H) \gamma_{n+1}(G)=K$, say, is a nontrivial abelian group with properties (a) and (b). Hence clearly $\beta_{p}(K)<K$ for some prime $p$ and so $\beta_{p}\left(\gamma_{n}(G)\right) \gamma_{n}(H) \gamma_{n+1}(G)<\gamma_{n}(G)$. Therefore it suffices to prove that

$$
\gamma_{n}\left(H \gamma_{2}(G) \beta_{p}(G)\right) \leqq \gamma_{n}(H) \gamma_{n+1}(G) \beta_{p}\left(\gamma_{n}(G)\right) .
$$

This is proved inductively (on $n$ ). The result is trivial for $n=1$. If $n>1$, suppose

$$
\gamma_{n-1}\left(H \gamma_{2}(G) \beta_{p}(G)\right) \leqq \gamma_{n-1}(H) \gamma_{n}(G) \beta_{p}\left(\gamma_{n-1}(G)\right),
$$

then 


$$
\begin{aligned}
\gamma_{n}\left(H \gamma_{2}(G) \beta_{p}(G)\right) & \leqq\left[\gamma_{n-1}(H) \gamma_{n}(G) \beta_{p}\left(\gamma_{n-1}(G)\right), H \gamma_{2}(G) \beta_{p}(G)\right] \\
& \leqq \gamma_{n}(H) \gamma_{n+1}(G) \beta_{p}\left(\gamma_{n}(G)\right)
\end{aligned}
$$

using (7) and (8) and

$$
\left[\gamma_{n-1}(G), \beta_{p}(G)\right] \leqq \beta_{p}\left(\gamma_{n}(G)\right) \gamma_{n+1}(G)
$$

and

$$
\left[\beta_{p}\left(\gamma_{n-1}(G)\right), G\right] \leqq \beta_{p}\left(\gamma_{n}(G)\right) \gamma_{n+1}(G)
$$

which follow from (10). This completes the proof.

\section{REFERENCES}

1. Marshall Hall, The theory of groups, Macmillan, New York, 1959.

2. Paul Hill, Remarks on a paper of Hobby and Wright, Proc. Amer. Math. Soc. 13 (1962), 80-81.

3. Charles Hobby and C. R. B. Wright, $A$ generalization of a theorem of $N$. Ito on p-groups, Proc. Amer. Math. Soc. 11 (1960), 707-709.

4. Zvonimir Janko, $A$ theorem on nilpotent groups, Glasnik Mat.-Fiz. Astronom. Drustvo mat. Fiz. Hrvatske II 15 (1960), 247-249.

The Australian National University, Canberra, Australia 